\title{
FACE RECOGNITION FOR SOCIAL MEDIA WITH Mobile Cloud Computing
}

\author{
Prasetyawidi Indrawan $^{1}$, Slamet Budiyatno ${ }^{2}$, Nur Muhammad Ridho ${ }^{3}$, and Riri \\ Fitri Sari ${ }^{4}$ \\ ${ }^{1,2,3,4}$ Department of Electrical Engineering, University of Indonesia, Depok, Indonesia \\ ${ }^{1}$ prasetyawidi.i@ui.ac.id \\ ${ }^{2}$ slamet.budiyatno@ui.ac.id \\ ${ }^{3}$ nur.muhammad82@ui.ac.id \\ ${ }^{4}$ riridui.ac.id
}

\begin{abstract}
Social Networking has become today's lifestyle and anyone can easily receive information about everyone in the world. It is very useful if a personal identity can be obtained from the mobile device and also connected to social networking. Therefore, we proposed a face recognition system on mobile devices by combining cloud computing services. Our system is designed in the form of an application developed on Android mobile devices which utilized the Face.com API as an image data processor for cloud computing services. We also applied the Augmented Reality as an information viewer to the users. The result of testing shows that the system is able to recognize face samples with the average percentage of $85 \%$ with the total computation time for the face recognition system reached 7.45 seconds, and the average augmented reality translation time is 1.03 seconds to get someone's information.
\end{abstract}

\section{KEYWORDS}

Cloud Computing, Social Network, Face Recognition, Face Detection, Augmented Reality

\section{INTRODUCTION}

Currently, social networking has become a very popular media for many people [1]. A lot of personal information can be obtained from this social network. Search for detailed-identity can be easily conducted through searching using the search engine sites or existing social networking website. However, this appears to be a less effective and the search must be performed in front of a computer or laptop. The development of mobile technologies such as smartphone and tablet allows a person to easily run a variety of multitasking activities including basic activities of the phones to run various applications such as email, multimedia, office applications, etc. In addition, there are a variety of services on the internet which is integrate their services with social network services. This condition was due to the current trend in it which is aimed the capability for sharing the core of social network. Today, most services on the internet must provide facilities for users to connect their services with a popular social network services, especially Facebook and Twitter. Start from this case, we designed a system that can connect a person with a variety of services through someone's identity.

Our proposed system uses a person's face as the primary identity. This is because in general, we know someone from his face. Our system has made facial identification to know the various social network and other internet activities followed by that person. By utilizing the camera

DOI : $10.5121 /$ ijccsa.2013.3102 
system on mobile devices, system identification can be done by a person using face recognition system. One thing to be considered in the application of facial recognition systems is face recognition system requires high computation. In this case, the mobile devices has only limited resources and there is a problem in implementing face recognition application in mobile devices[2].

The work of $[3,4]$ show the advantage of the computing process of facial recognition systems which is done outside the server (cloud computing). The implementation of cloud computing technology on mobile devices is aimed at creating effective computation on mobile devices for performing process of face recognition.

In this work, the problem of interest is the design and implementation of face recognition module on mobile devices associated with the process of cloud computing. The design and implementation is done by making an application to perform face detection system on Android mobile device (onloading) including augmented reality module and performing face recognition module using cloud's services.

\section{LITERATURE REVIEW}

This section will describe the explanation of the face recognition system, cloud computing technology, Face.com API, and augmented reality concept.

\subsection{Face Recognition}

Face recognition system is a system that performs engineering method in an image to search for the identity or the information contained in an image. Facial recognition systems are generally divided into two stages [5]. The first stage is the face detection module which is the early stage (pre-processing). Then, is followed by the facial recognition stage. Several techniques that can be used to detect face in an image are [6]:

a. Knowledge-based method

b. Feature invariant approaches

c. Template matching methods

d. Appearance based methods

Meanwhile, the face recognition system is a system designed on a computer. This system is created to help identifying a person's face through the image or digital video. One of the method commonly used in the face recognition system is a way to compare the facial feature of an image with a database of faces that have been taken earlier. This is shown in Figure 1, which describes the flow of the process of facial recognition. 


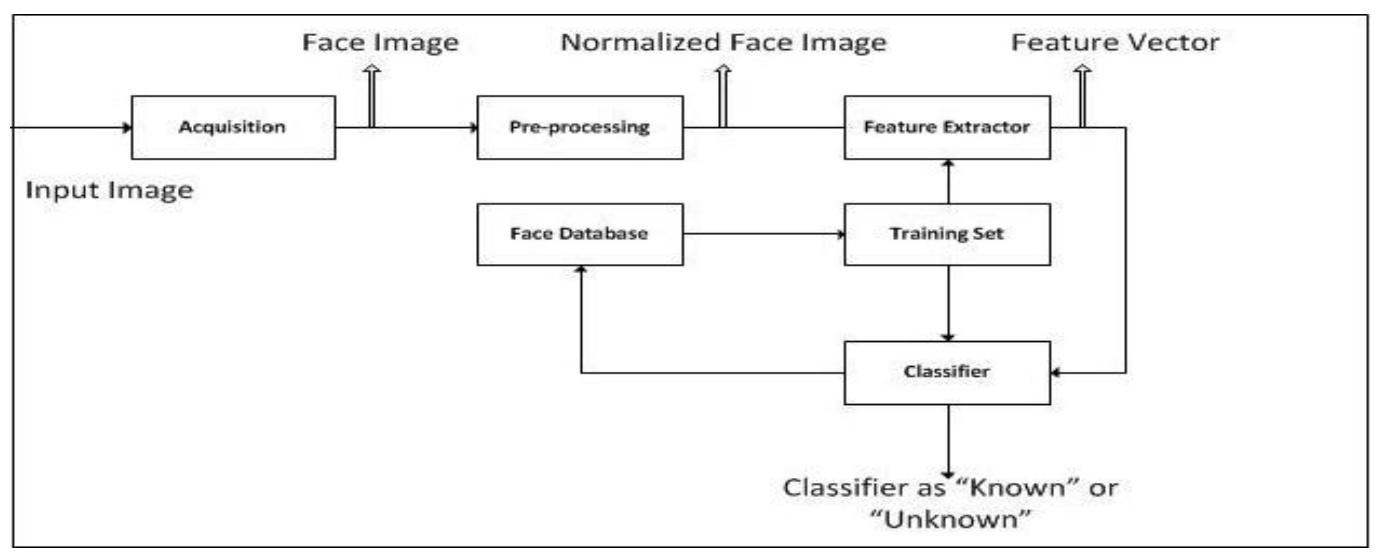

Figure 1 Face Recognition Workflow [7]

\subsection{Cloud Computing Technology}

Cloud computing system is a combination of computer technology as a processor utilization and development of internet-based computing in which information technology-related capabilities provided as a service or on demand [8]. One of the cloud computing service is Google App Engine (GAE). GAE is a Platform as a Services in cloud computing for building and hosting our web-based applications in Google data center [9]. Applications will be built on a multiplevirtualized servers. Google App Engine provides an on-demand services, that the resource will be used according to the need of users. GAE has an automatic system to adjust resource for applications that are built to increase the existing demand.

\subsection{Face.com API}

Face.com is an Application Programming Interface (API) services for face recognition process. Face.com can be classified into PaaS cloud computing service [10]. Face.com provides an API for developers to develop software that implement a face recognition function. The process of facial recognition with Face.com API as well as face recognition process in general. First, the user must perform training to the new faces. In this training process, there is a face detection stage and the result will be saved. Once the face has successfully trained, the face can be recognized by the system.

\subsection{Augmented Reality}

Augmented Reality has been there for a long time, but has been established as a research area in 1990's. There are many definitions of augmented reality, however the general assumption is that the augmented reality enables an enriched perspective by superimposing virtual objects on the real world in a way that persuades the viewer that the virtual object is a part of the real. Therefore, augmented reality is a crossover between the real and virtual world. Generally, augmented reality systems are divided into two types :

\section{a. Augmented reality based on marker.}

A method that utilizes an illustration of a black marker and a square-shaped anthers with a thick black border and white background. Through the position faced with a computer camera, the computer will make the process of creating $2 \mathrm{D}$ or $3 \mathrm{D}$ virtual world. 


\section{b. Augmented reality without marker (markerless AR).}

AR method does not require a marker to show the elements of the virtual world when combined with a real-world environment. The use of the markerless method is commonly used for face tracking, object tracking and 3D motion tracking.

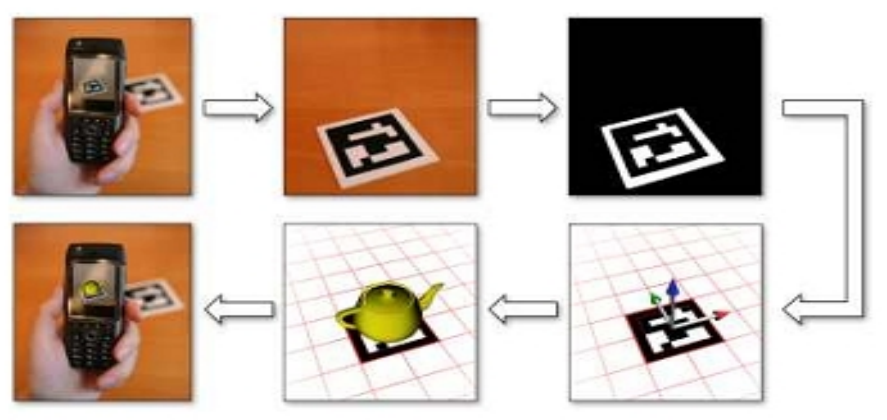

Figure 2 Augmenting virtual object in the real world image [13]

Figure 2 depicted the process of combining real world and virtual object for marker based AR. Since the begining of Augmented Reality (AR) systems, the potential of collaborative AR was exploited for different activities such as in military, in football match or in the helmet of the pilot. They can see several pieces of information. Nowadays some elements of AR are used for mobile phone applications $[14,15]$. By just pointing the camera to an object, we will immediately receive information about the object on the screen. In this paper, we implement augmented reality without marker on Android platform, but we use the face as a primary marker for augmented reality display.

\section{Proposed Method}

The system in this paper was designed to combine the computation which is run on mobile device and the advantage of cloud computing as explained in [4]. The computation on mobile device (onloading) will perform the face detection module and augmented reality to interact with the user. The other computation will run in the cloud server (offloading) using Google App Engine (GAE) dan Face.com service. Actually, the system in this paper was design in three main modules including face detection on Android mobile device, face recognition on cloud server using GAE and Face.com API, and augmented reality as a result of a face recognition module on mobile device. The overall system design is shown in Figure 3. 


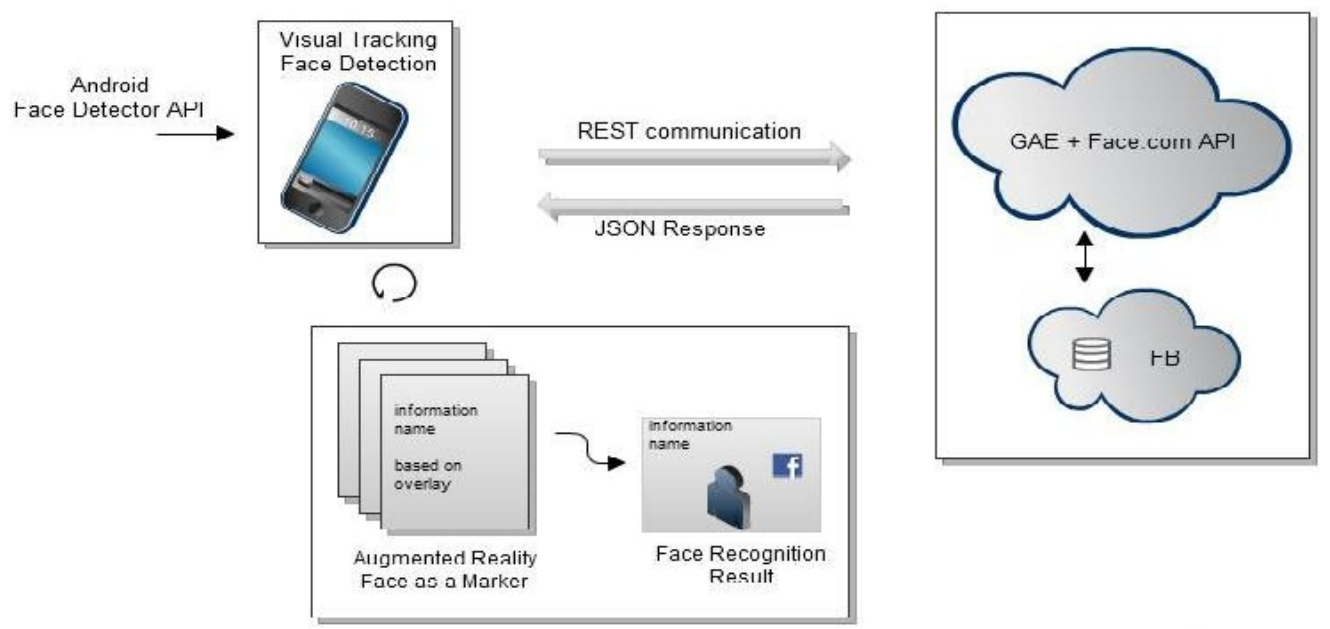

Figure 3 Block Diagram of System

Firstly, the system will perform a face detection through mobile device (onloading). The face detection itself, will use native API from Android called Android Face Detector API that detect face from a bitmap. Then, the application on mobile device will make the process of video stream which is directed to the face object based on the operations selected by the user. After face detected, mobile device will crop the image only on the face aspect as described in Figure 4. The algorithm for cropping the face image is as follows : Get the mid points of all the faces in the image, the confidence value should be higher than 0.4 [11]; Calculate a rectangle around the face which is about the dimensions as shown in Figure 4, the distance between the eyes is A ; Then, crop the image from the coordinates that have just been calculated.

After that, the results of the face detection will be processed for the offloading of the face recognition process to the cloud server by REST communication [12] in the data network. Figure 5 shows the sequence diagram of this system.

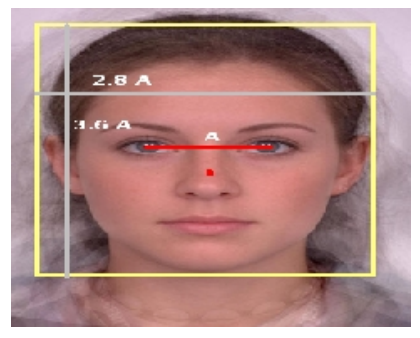

Figure 4 Illustration of Cropping Face Image 
International Journal on Cloud Computing: Services and Architecture (IJCCSA),Vol.3, No.1, February 2013

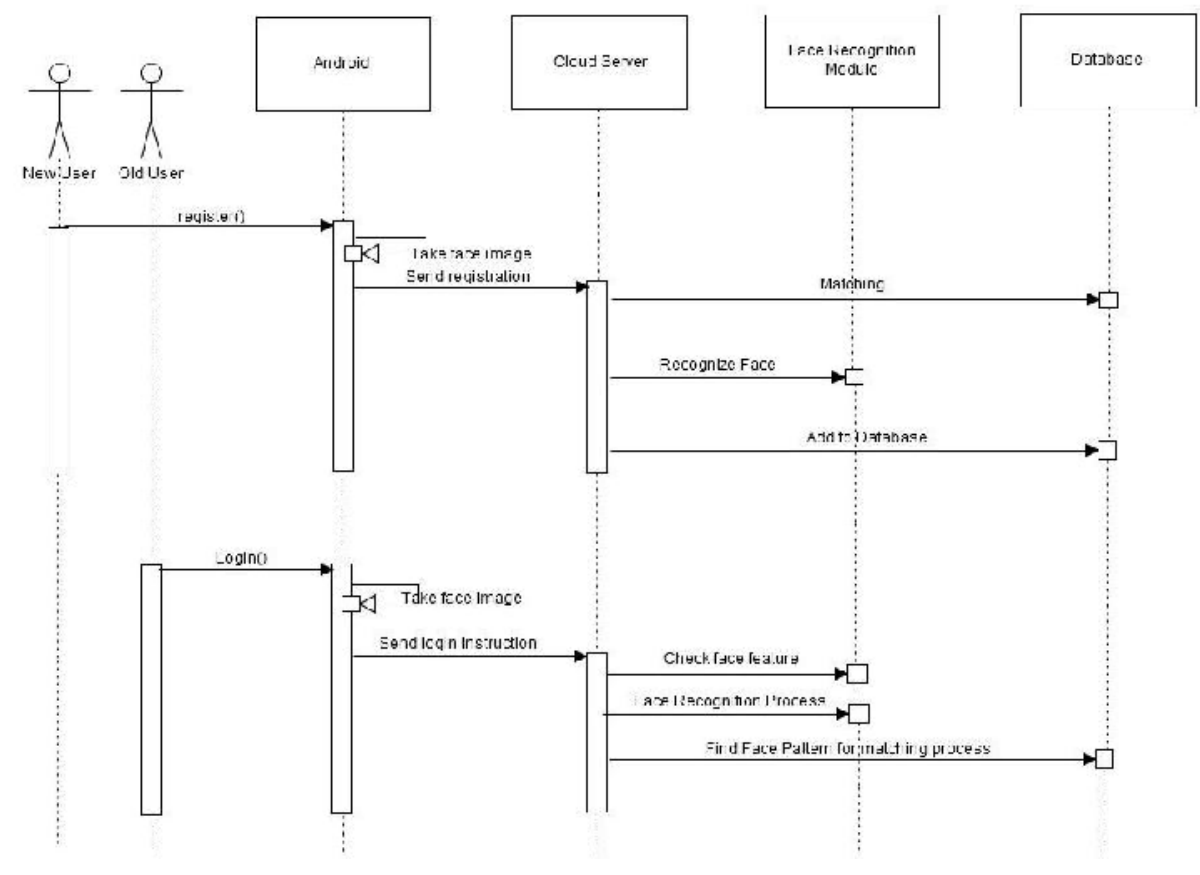

Figure 5 Sequence Diagram of System

The next step for this system is to perform face recognition module on the cloud server. Figure 6 shows the block diagram of the face recognition module. There are two Python scripts in the face recognition module. Main.py is a script which has a function as a connector with Android devices and also for Blobstore services caller. Face_client.py is a script which has a function as an API caller to the Face.com server. When the cloud server got the image, the face image will be proceed to know the identity of the face image. After the cloud server recognized the face image, it will return the result to the mobile device with json response which include, personal identity of the person. Then, mobile device will decode the json response and show the result as an augmented reality.

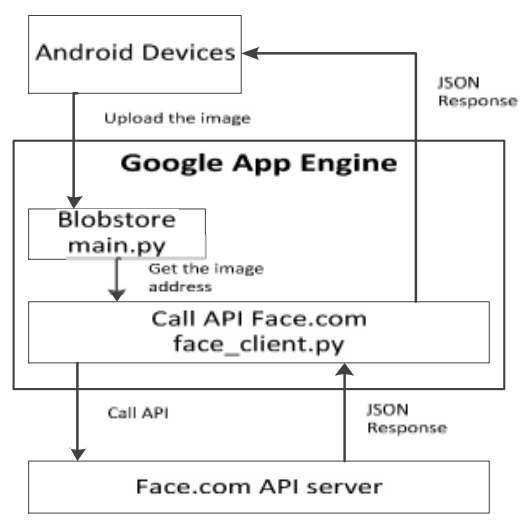

Figure 6 Block Diagram of Face Recognition Module 


\section{RESULT AND ANALYSIS}

There are three main modules which has been tested from this work i.e face detection module, face recognition module, and augmented reality module. The result and analysis will be described in the following sub-sections.

\subsection{Face Detection}

Face detection module in the mobile device is the first stage in the main functionality of this face recognition application. The process of face detection module work as a process of onloading that run individually on the mobile device. The technical testing done in this module consists of testing the camera resolution variations. Testing of the variation is done by varying resolution's camera ranging from low to high. The results of these variations in the resolution of the camera that would be material to know the time required to perform the face detection process in an image. Tests conducted in the face detection module will be performed 10 times for each condition of the camera resolution. The result of this testing will be shown in Figure 7 that also implemented augmented reality concept.
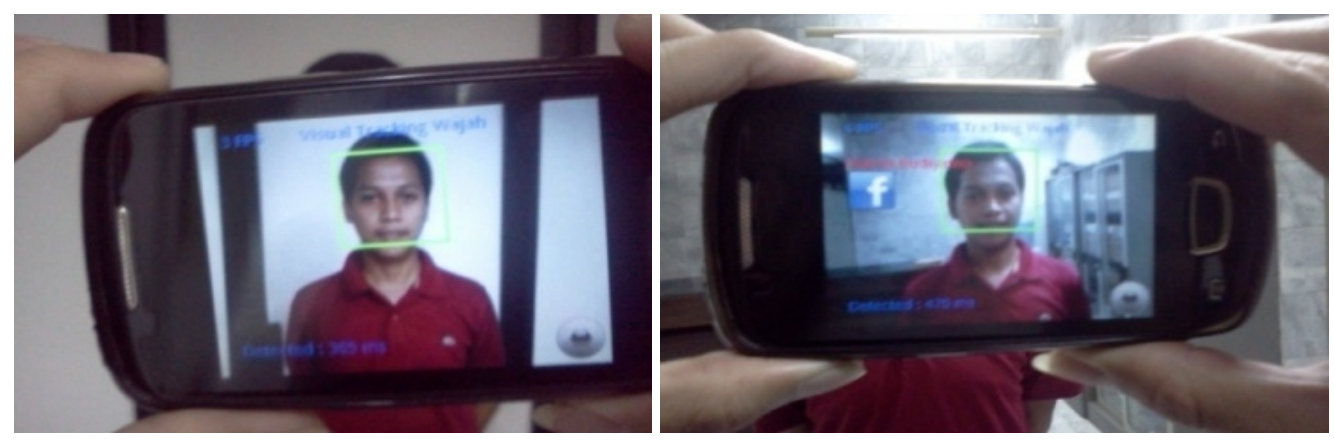

Figure 7 The Results of Face Detection Using Augmented Reality concept.

The test results of face detection system for mobile devices are implemented in two type that used low class (Galaxy GT S5570) with a $600 \mathrm{MHz}$ CPU specs, $384 \mathrm{Mb}$ RAM, and quality of 3.15 megapixel camera and middle class (W I8150 Galaxy) which has the specification of $1.4 \mathrm{GHz}$ CPU, $512 \mathrm{Mb}$ RAM, and quality 5 megapixel camera. When the testing process, it was found that the working time of a face detection system in mobile devices experience the difference in each variation of the camera resolution. This is because the amount of work is a growing field that is marked with greater resolution. When using a resolution of 240x160 pixels, the system can recognize the presence or absence of the face with an average time of 0.46 seconds. When the resolution was increased to $320 \times 240$ pixels, face detection system in mobile devices requires a longer working time. Greater use of the resolution would affect the long absence of the processing time of the face detection system. The processor speed also affect the working of the face detection system on mobile devices. The difference can clearly be seen in Figure 8. 


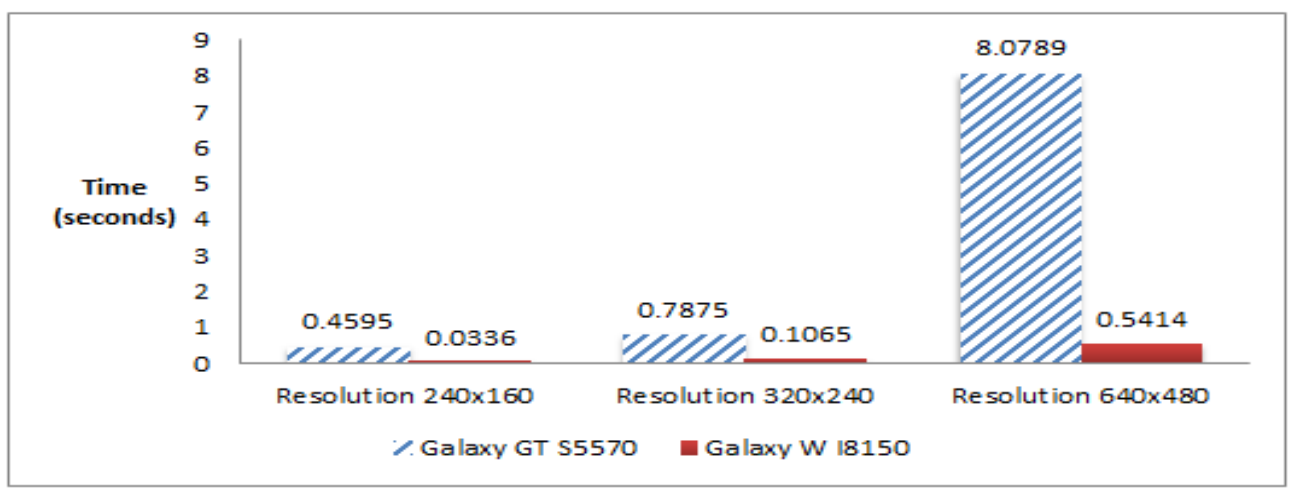

Figure 8 Face Detection System With Variation of the Camera Resolution

\subsection{Face Recognition}

The results of the face recognition that has been built will be discussed in this chapter as shown in Figure 9. This test aimed at obtaining an analysis of the level of success in the system to perform its function. The number of face data used in this test is 50 faces, with details of three men and two women with 10 face each data. Examples of faces used in this testing are presented in Table 1. In the tests performed with the variation of the number of training data, we obtained the results as shown in Table 2.

Table 1 Face Samples

\begin{tabular}{|l|l|l|l|l|l|}
\hline Face Object & Object 1 & Object 2 & Object 3 & Object 4 & Object 5 \\
\hline Face Image & & (2) & & & \\
& & & & \\
\hline
\end{tabular}

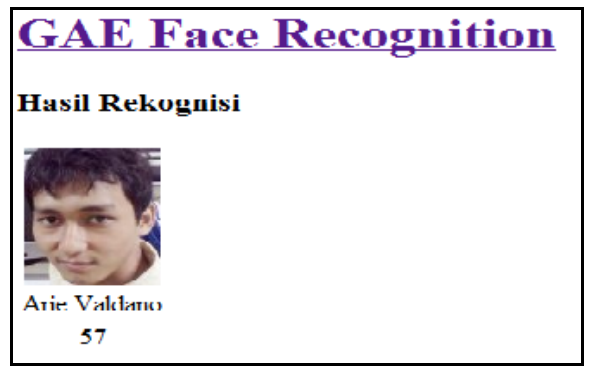

Figure 9 Result of Recognition Process on GAE

Figure 10 shows the effect of the number of training data on the level of face recognition of our system. $\mathrm{X}$-axis show the number of training data, while the $\mathrm{Y}$ axis expresses the degree of face recognition. With the number of training data is only one piece, the recognition rate remains low at $20 \%$. Along with the increasing number of the training data, it increased the level of recognition. When the training data of five pieces, the recognition rate reached the average of $100 \%$ from 10 trials. Figure 11 shows the effect of the number of data training to the result of error rates. 
International Journal on Cloud Computing: Services and Architecture (IJCCSA),Vol.3, No.1, February 2013

Table 2 Test Result with Variation of the Number of the Training Data

\begin{tabular}{|c|c|c|}
$\begin{array}{c}\text { The Number of } \\
\text { Data Training }\end{array}$ & $\begin{array}{c}\text { Recognition } \\
\text { Rate (\%) }\end{array}$ & $\begin{array}{c}\text { Error } \\
\text { Rate (\%) }\end{array}$ \\
\hline $\mathbf{1}$ & 20 & 33 \\
& 60 & 31.2 \\
\hline $\mathbf{3}$ & 80 & 29.2 \\
\hline $\mathbf{4}$ & 90 & 2.8 \\
\hline $\mathbf{5}$ & 100 & 31.2 \\
\hline $\mathbf{6}$ & 100 & 30 \\
\hline $\mathbf{7}$ & 100 & 30.2 \\
\hline $\mathbf{8}$ & 100 & 30.2 \\
\hline $\mathbf{9}$ & 100 & 30.2 \\
\hline $\mathbf{1 0}$ & 100 & 31.6 \\
\hline Average & $\mathbf{8 5}$ & $\mathbf{3 0 , 4 8}$ \\
\hline
\end{tabular}

In contrast to the recognition rate, error rate is obtained from the variation of the number of training data which has a quite different value. With only single training data, the error rate is obtained at a value of $33 \%$. The error values continue to decrease until the training data is 4 , with a value of $28 \%$ error. But when the number of training data continues to be increased, the error rate to be increase to $31.2 \%$. After that the error will be returned to the stability value of $30.2 \%$.

The differences in the recognition rate and error rate, indicated that the number of training data affects the value of recognition and error rate. When there are only few training data, the learning system of the face will low. The more training data, the better learning system.

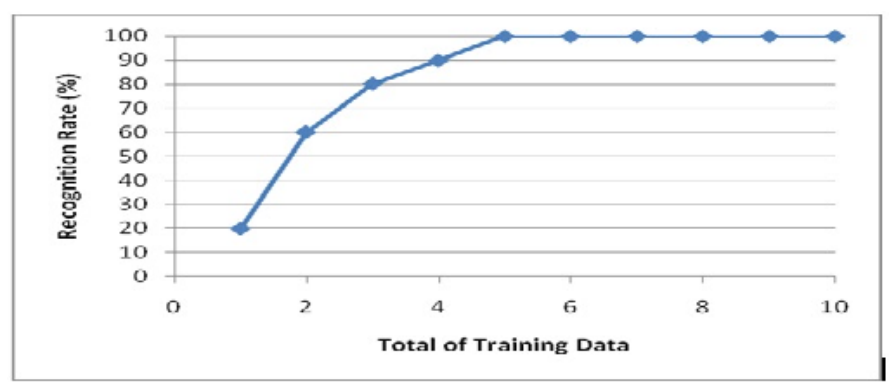

Figure 10 The Effect of Total Training Data for Face Recognition Effect

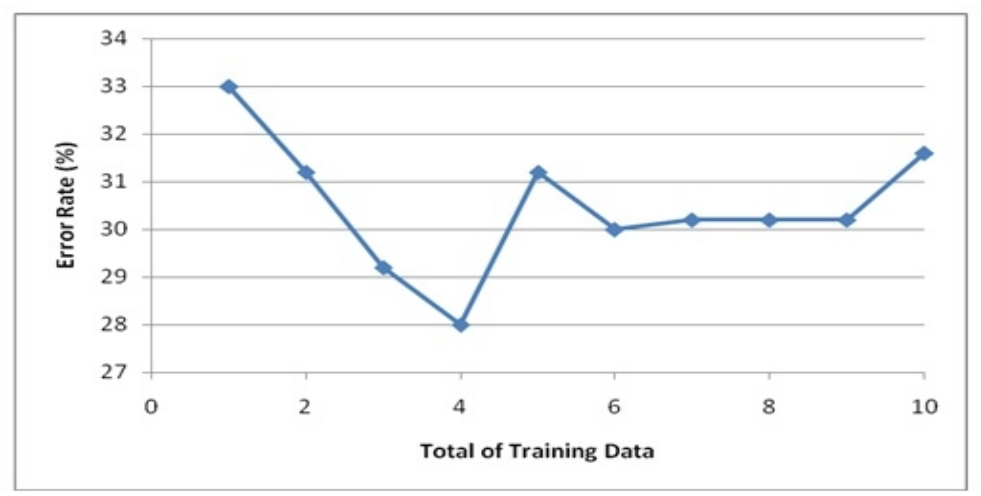

Figure 11 The Effect of Total Training Data for Face Recognition Error Rate 


\subsection{Augmented Reality}

This section we discuss the result of testing and analysis of our system. The systems has been successfully developed on android devices. The functionality of the whole system has been working well. We tested to find out the weakness and error. Testing was intended the system to find out the translation time of augmented reality. Tests performed on normal lighting conditions using ten samples and performed on the front face recognition process as many as 10 attempts for each face with different expression. It is intended to maximize the face recognition results obtained and to get more detailed information on the face intended to be recognized. The average time to test the result of the translation is shown in Figure 12.

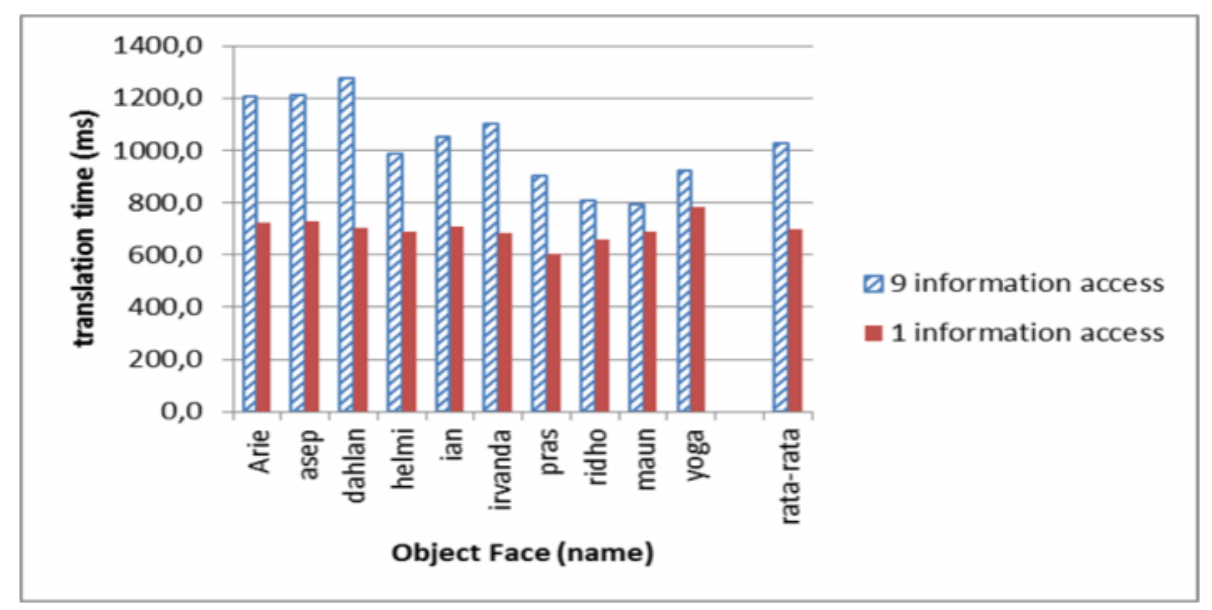

Figure 12 Average Translation Time

Based on the translation of the image obtained from 9 information access, information that contains the complete data about the owner of the familiar faces are much slower compared with an access permission to display bio information. In displaying detailed information the translation time lasts for 1.03 seconds, while the bio-data to show the translation time is 0.69 seconds. This happens because the system works by making the initial approach of face detection and face recognition. Condition that occurs when the test was to ignore the value of face recognition are issued either agree or disagree with the input face image. Determination of the face affects the process of facial recognition as the face detection results will serve as the primary marker in our recognition. If there are objects that are blocking the area of the face like a scarf or hair covering the forehead, the face recognition algorithm will not deliver good result as the translation was not the same for each time. The result of translation that interact with social media is shown in Figure 13.
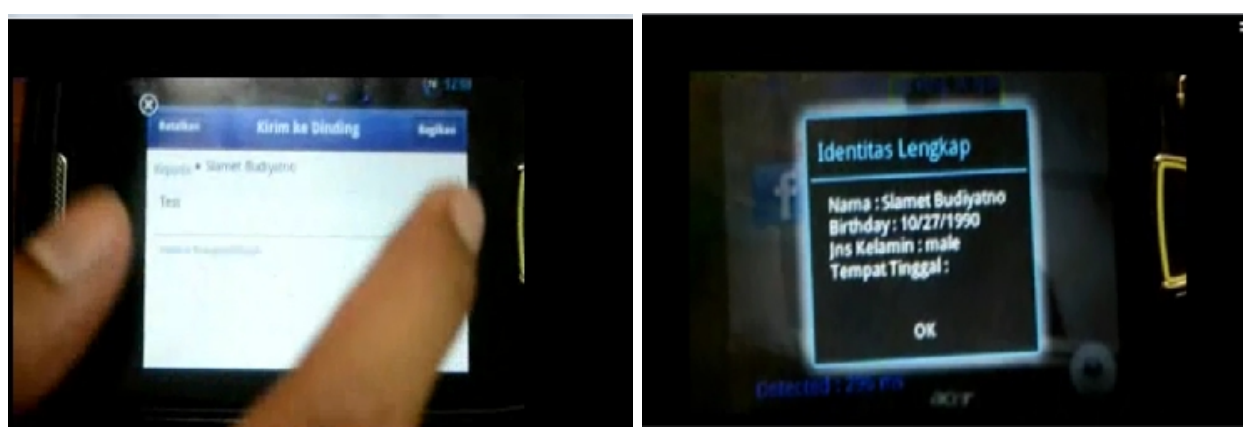

Figure 13 Illustration of Social Media Interaction 
In addition to the effect caused by the recognition process, it should be noted that the information will be displayed in the different request. Plain text files take longer to be parsed and translated. JSON translation to spend a long time, to exchange data with complete information requests because of size issue. JSON object with complete information has a size greater than JSON with a little information. The different is around a hundred bytes. This impact on the variation of translation time.

\subsection{Total Computation Time}

This section explains the testing result of all modules that implemented on mobile devices. This section discussed about the computing process which was done on the face detection module and the face recognition module. Computational processes that occur in the face recognition systems shows the effectiveness of the, especially when integrated with cloud computing technology. The application of cloud computing technology to make the process of offloading can be used to save energy. Here is an overall result of the computation process of facial recognition systems on mobile devices as shown in Table 3.

Table 3 Description of Computational Time for Face Recognition System on Mobile Devices

\begin{tabular}{|l|c|c|c|c|c|c|}
\hline Mobile Type & \multicolumn{3}{|c|}{ GTS5570 } & \multicolumn{3}{c|}{ W I8150 } \\
\hline $\begin{array}{l}\text { Resolutions } \\
\text { (pixels) }\end{array}$ & $240 \times 160$ & $320 \times 240$ & $640 \times 480$ & $240 \times 160$ & $320 \times 240$ & $640 \times 480$ \\
\hline $\begin{array}{l}\text { Face Detection } \\
\text { (onloading) }\end{array}$ & $0.4595 \mathrm{~s}$ & $0.7875 \mathrm{~s}$ & $8.0789 \mathrm{~s}$ & $0.0336 \mathrm{~s}$ & $0.1065 \mathrm{~s}$ & $0.5414 \mathrm{~s}$ \\
\hline $\begin{array}{l}\text { Face Recognition } \\
\text { (offloading) }\end{array}$ & $7 \mathrm{~s}$ & $7 \mathrm{~s}$ & $7 \mathrm{~s}$ & $7 \mathrm{~s}$ & $7 \mathrm{~s}$ & $7 \mathrm{~s}$ \\
\hline $\begin{array}{l}\text { Total } \\
\begin{array}{l}\text { Computation } \\
\text { Time }\end{array}\end{array}$ & & & & & & \\
\hline
\end{tabular}

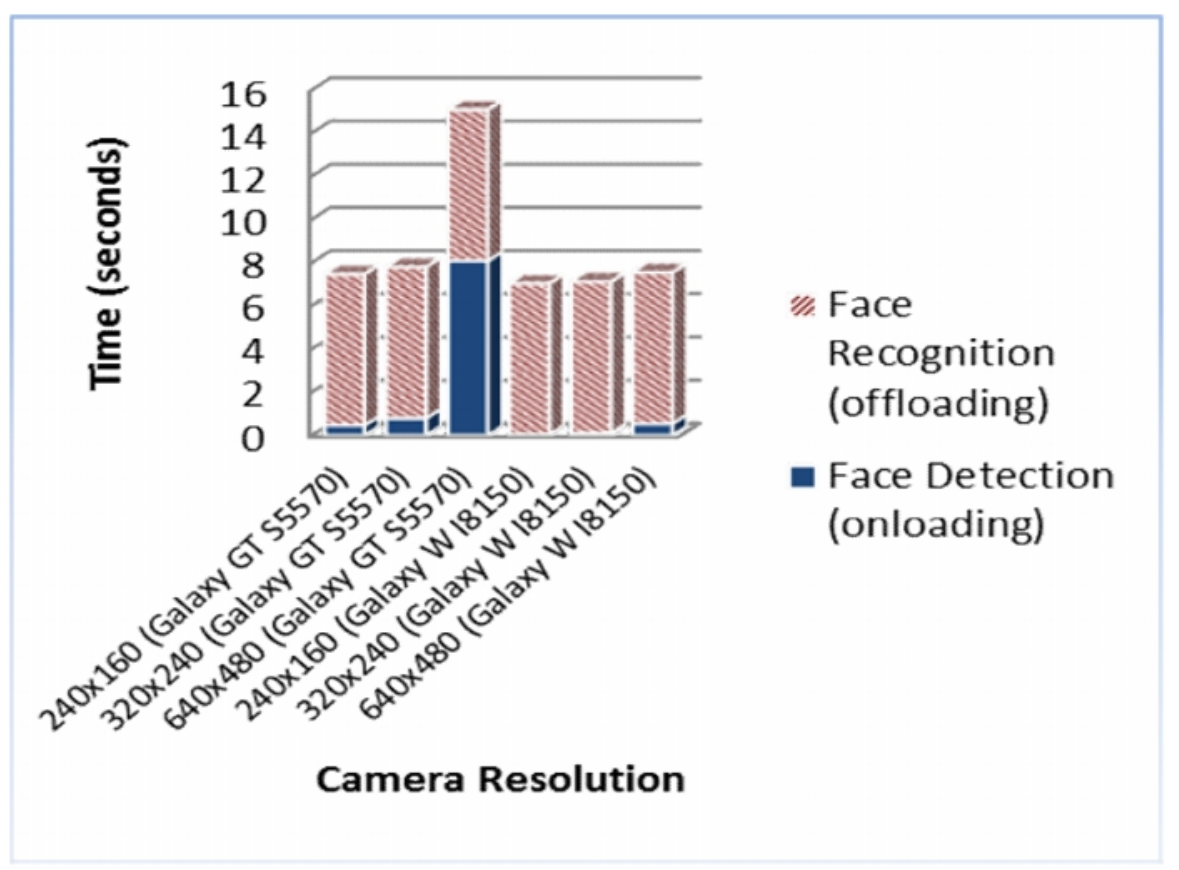

Figure 14 Total Computation Time for Different Device 
Figure 14 shows that the computation to perform the face detection process (onloading) require a faster time on all types of mobile devices. This differs from the process of offloading that requires a longer computation time. This is due to the process of offloading the server cloud computing requires high computation and the process of sending the result of face recognition which is also highly dependent on network connectivity. However, the overall process of face recognition is implemented on mobile device which indicate the results are not so bad. The process of visual tracking or face detection process that has been tested run well and indicates the system works in real-time.

\section{Conclusions}

In this work, we implemented a real-time image processing application especially the face recognition system and user interface on Android mobile device. We also created augmented reality application on this project to provide information about the recognizable faces viewed by the user. The result of testing indicates that face recognition rate reached the average percentage of $85 \%$. The use of face recognition system based on Augmented Reality provides an attractive interface for the user. From the test results, the translation time is 1.03 seconds to show the augmented reality.

Face recognition system has been implemented using cloud computing technology (offloading process) that uses REST to the cloud server communications which are quite satisfactory with a fairly accurate result. However, the overall system do not represent a real time system because 7 seconds is too long. For future development, the application of cloud computing technology can be considered as an alternative to save on computing in mobile devices along with its development is quite extensive. So, the future, this system should be capable of being a face recognition system in a real time as the development of the communications network in providing a faster access.

\section{REFERENCES}

[1] Ashraf Jalal. "The Use of Social Networking in Education : Challenge and Opportunities. WCSIT. 2012.

[2] N. Balasubramanian, A Balasubramanian, and A. Venkataramani, "Energy Consumption in Mobile Phone : a measurement study and implications for network applications,"ACM. 2009.

[3] A.P. Miettinen and J.K. Nurminen, "Energy efficiency of mobile clients in cloud computing," HotCloud 2nd USENIX Workshop on Hot Topics in Cloud Computing, 2010.

[4] K.Kumar and Y.H. Lu, "Cloud computing for mobile users: Can offloading computation save energy". IEEE Computer Society, vol. 43, no. 4, 2010.

[5] Face Recognition, http://www.shervinemami.co.cc/faceRecognition.html retrieved December 18, 2011.

[6] D. Kriegman Ming-Hsuan Yang and N. Ahuja, "Detecting faces in images: A survey," IEEE Transactions on Pattern Analysis and Machine Intelligence, vol. 24, no. 1, pp. 34-58, January 2002.

[7] P.Viola and M. Jones. "Robust Real Time Face Detection". International Journal of Computer Vision 57, 137-154. 2004.

[8] Rajkumar Buyya, James Broberg, Andrzej Goscinski. "Cloud Computing Principle and Paradigm" Wiley, 2011.

[9] Google App Engine, http://code.google.com/appengine/docs/whatisgoogleappengine.html, retrieved November 2nd, 2011.

[10] Face API, http://developers.face.com, retrieved April 1st 2012.

[11] Developer Android, http://developer.android.com/, retrieved December 18th, 2011

[12] REST Communication ,http://www.ibm.com/developerworks/webservices/library/ws-restful/ retrieved May 31, 2012 
[13] Daniel Wagner and Dieter Schmalstieg. Artoolkitplus for pose tracking on mobile devices. Proceedings of 12th Computer Vision Winter Workshop, page 139-146, Graz University of Technology, Institute for Computer Graphics and Vision, February 2007.

[14] Kim, Jaeyoung \& Jun, Heesung, "Implementation of Image Processing and Augmented Reality Programs for Smart Mobile Device", IEEE The 6th International Forum on Strategic Technology, Korea, 2011.

[15] Maoqiang Song, Wenkuo Xiong, Xiangling Fu, "Research on Architecture of Multimedia" in Proc. Of Internet Technology and Applications, 2010.

\section{AUTHORS}

Prasetyawidi Indrawan

Graduated from the Undergraduate Program in Computer Engineering, Department of Electrical Engineering, Faculty of Engineering, University of Indonesia

Slamet Budiyatno

Graduated from the Undergraduate Program in Computer Engineering, Department of Electrical Engineering, Faculty of Engineering, University of Indonesia

Nur Muhammad Ridho

Graduated from the Undergraduate Program in Computer Engineering, Department of Electrical Engineering, Faculty of Engineering, University of Indonesia

\section{Riri Fitri Sari}

Professor of Computer Engineering, Department of Electrical Engineering Faculty of Engineering, University of Indonesia

She receive a PHD in Multimedia Network at the School of Computing, University of Leeds and MSc from Department of Computing, The University of Sheffield, UK.

Dr. Sari is a senior member of IEEE
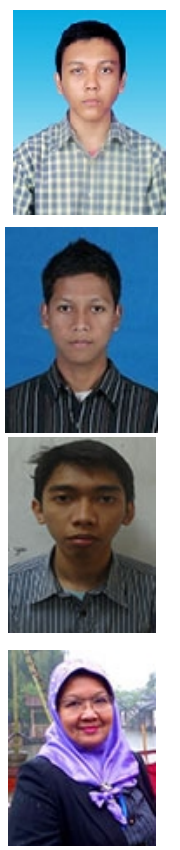